\title{
Connectedness of approximate solutions set for vector equilibrium problems in Hausdorff topological vector spaces
}

\author{
Bin Chen ${ }^{1}$, Qing-you Liu², Zhi-bin Liư ${ }^{3}$ and Nan-jing Huang ${ }^{1 *}$
}

\author{
* Correspondence: \\ nanjinghuang@hotmail.com \\ 'Department of Mathematics, \\ Sichuan University, Chengdu, \\ Sichuan 610064, P.R. China \\ Full list of author information is \\ available at the end of the article
}

\begin{abstract}
In this paper, a scalarization result of $\varepsilon$-weak efficient solution for a vector equilibrium problem (VEP) is given. Using this scalarization result, the connectedness of $\varepsilon$-weak efficient and $\varepsilon$-efficient solutions sets for the VEPs are proved under some suitable conditions in real Hausdorff topological vector spaces. The main results presented in this paper improve and generalize some known results in the literature.
\end{abstract}

Keywords: vector equilibrium problem, scalarization method, $\varepsilon$-weak efficient solution, $\varepsilon$-efficient solution, connectedness

\section{Introduction}

Let $K$ be a nonempty subset of a real Hausdorff topological vector space $E$, and $f: K \times$ $K \rightarrow R$ a bifunction such that $f(x, x) \geq 0$ for all $x \in K$. Then, the scalar equilibrium problem consists in finding $\bar{x} \in K$ such that

$$
f(\bar{x}, y) \geq 0, \quad \forall y \in K \text {. }
$$

It provides a unifying framework for many important problems, such as optimization problems, variational inequality problems, complementary problems, minimax inequality problems, Nash equilibrium problems, and fixed point problems, and has been widely applied to study problems arising in economics, mechanics, and engineering science (see [1]). On the other hand, several operations research problems are formulated with a multicriteria consideration. These are vector optimization problems, vector variational inequality and complementarity problems and vector equilibrium problems (VEPs). Recently, the VEP has received much attention by many authors because it provides a unified model including vector optimization problems, vector variational inequality problems, vector complementarity problems and vector saddle point problems as special cases (see, for example, [2-24] and the references therein).

It is well known that another important problem for VEPs is to study the topological properties of solutions set. Among its topological properties, the connectedness is of interest. Recently, Lee et al. [25], Cheng [26] have studied the connectedness of weak efficient solutions set for vector variational inequalities in finite dimensional Euclidean space. Gong [27-29] has studied the connectedness of the various solutions set for VEPs in infinite dimension space. Chen et al. [30] studied the connectedness and the

\section{SpringerOpen ${ }^{\bullet}$}

(C) 2011 Chen et al; licensee Springer. This is an Open Access article distributed under the terms of the Creative Commons Attribution License (http://creativecommons.org/licenses/by/2.0), which permits unrestricted use, distribution, and reproduction in any medium provided the original work is properly cited. 
compactness of the weak efficient solutions set for set-valued VEPs and the set-valued vector Hartman-Stampacchia variational inequalities in normed linear space. Gong and Yao [31] have studied the connectedness of the set of efficient solutions for generalized systems. Zhong et al. [32] have studied the connectedness and path-connectedness of solutions set for symmetric VEPs. However, the connectedness of approximate solutions set for VEPs remained unstudied.

In this paper, we show a scalarization result of $\varepsilon$-weak efficient solution for a VEP. Using this scalarization result, we discuss the connectedness of $\varepsilon$-weak efficient and $\varepsilon$-efficient solutions sets for VEPs under some suitable conditions in real Hausdorff topological vector spaces. The main results presented in this paper generalize some known results due to Gong [27] and Gong and Yao [31].

\section{Preliminaries}

Throughout this paper, let $X$ and $Y$ be two real Hausdorff topological vector spaces and $A$ a nonempty subset of $X$. Let $F: A \times A \rightarrow Y$ be a mapping and $C$ be a closed convex pointed cone in $Y$. The cone $C$ induces a partial ordering in $Y$, defined by

$$
z_{1} \leq z_{2} \quad \text { if and only if } z_{2}-z_{1} \in C .
$$

Let

$$
C^{*}=\left\{f \in Y^{*}: f(y) \geq 0 \quad \text { for all } y \in C\right\}
$$

be the dual cone of $C$.

Denote the quasi-interior of $C^{*}$ by $C^{\#}$, that is,

$$
C^{\#}=\left\{f \in Y^{*}: f(y)>0 \quad \text { for all } y \in C \backslash\{0\}\right\} .
$$

Let $D$ be a nonempty subset of $Y$. The cone hull of $D$ is defined as

$$
\text { cone }(D)=\{t d: t \geq 0, d \in D\} \text {. }
$$

A nonempty convex subset $B$ of the convex cone $C$ is called a base of $C$ if

$$
C=\text { cone }(B) \text { and } 0 \notin c l(B) \text {. }
$$

It is easy to see that $C^{\#} \neq \varnothing$ if and only if $C$ has a base.

Throughout this paper, we always assume that int $C \neq \varnothing$. Let $e$ be a fixed point in $\operatorname{int} C$ and we set

$$
\begin{gathered}
C^{\prime}=\left\{f \in C^{*} \backslash\{0\}: f(e)=1\right\}, \\
C^{\prime \prime}=\left\{f \in C^{\#}: f(e)=1\right\} .
\end{gathered}
$$

Now, we give the concepts of $\varepsilon$-weak efficient solution, $\varepsilon$-efficient solution, and $\varepsilon-f$ efficient solution for the VEP.

Definition 2.1. A vector $x \in A$ satisfying

$$
F(x, y) \notin-i n t C-\varepsilon e \text { for all } y \in A \text {, }
$$

is called a $\varepsilon$-weak efficient solution to the VEP. Denote by $V_{\varepsilon-W}(A, F)$ the set of all $\varepsilon$ weak efficient solutions to the VEP.

Definition 2.2. A vector $x \in A$ satisfying

$$
F(x, y) \notin-C \backslash\{0\}-\varepsilon e \quad \text { for all } y \in A,
$$


is called a $\varepsilon$-efficient solution to the VEP. Denote by $V_{\varepsilon}(A, F)$ the set of all $\varepsilon$-efficient solutions to the VEP.

Definition 2.3. Let $f \in C * \mid\{0\}$. A vector $x \in A$ is called a $\varepsilon-f$ efficient solution to the VEP if

$$
f(F(x, y)) \geq-\varepsilon \text { for all } y \in A,
$$

Denote by $V_{\varepsilon-f}(A, F)$ the set of all $\varepsilon-f$ efficient solutions to the VEP.

Definition 2.4. [33] Let $G$ be a set-valued map from a topological space $W$ to another topological space $Q$. We say that $G$ is

(i) upper semicontinuous at $x_{0} \in W$ if, for any neighborhood $U\left(G\left(x_{0}\right)\right)$ of $G\left(x_{0}\right)$, there is a neighborhood $U\left(x_{0}\right)$ of $x_{0}$ such that $G(x) \subset U\left(G\left(x_{0}\right)\right)$ for all $x \in U\left(x_{0}\right)$;

(ii) upper semicontinuous on $W$ if it is upper semicontinuous at each $x \in W$;

(iii) lower semicontinuous at $x_{0} \in W$ if, for any net $\left\{x_{\alpha}: \alpha \in I\right\}$ converging to $x_{0}$ and for any $y_{0} \in G\left(x_{0}\right)$, there exists a net $y_{\alpha} \in G\left(x_{\alpha}\right)$ that converges to $y_{0}$;

(iv) lower semicontinuous on $W$ if it is lower semicontinuous at each $x \in W$;

(v) continuous on $W$ if it is both upper semicontinuous and lower semicontinuous on $W$;

(vi) closed if $\operatorname{Graph}(G)=\{(x, y): x \in W, y \in G(x)\}$ is a closed subset in $W \times Q$.

\section{Scalarization}

Lemma 3.1. Suppose $F(x, A)+C$ is a convex set for each $x \in A$. Then,

$$
V_{\varepsilon-W}(A, F)=\bigcup_{f \in C^{\prime}} V_{\varepsilon-f}(A, F) .
$$

Proof. We first prove that

$$
\bigcup_{f \in C^{\prime}} V_{\varepsilon-f}(A, F) \subset V_{\varepsilon-W}(A, F) .
$$

In fact, letting $x_{0} \in \bigcup_{f \in C^{\prime}} V_{\in-f}(A, F)$, then there exists $f \in C^{\prime}$,

$$
f\left(F\left(x_{0}, y\right)\right) \geq-\varepsilon, \quad \text { for all } y \in A .
$$

We claim that $x_{0} \in V_{\varepsilon-W}(A, F)$. If not, then there exists $y_{0} \in A$ such that $F\left(x_{0}, y_{0}\right) \in$ -int $C-\varepsilon e$. Thus, we have

$$
f\left(F\left(x_{0}, y_{0}\right)+\varepsilon e\right)<0
$$

and so

$$
f\left(F\left(x_{0}, y_{0}\right)\right)<-f(\varepsilon e)=-\varepsilon,
$$

which is a contradiction to (3.1).

We next prove that

$$
V_{\varepsilon-W}(A, F) \subset \bigcup_{f \in C^{\prime}} V_{\varepsilon-f}(A, F) .
$$

Let $x \in V_{\varepsilon-W}(A, F)$. Then, $F(x, A) \cap(-\operatorname{int} C-\varepsilon e)=\varnothing$. Since $C$ is a pionted convex cone, we have

$$
(F(x, A)+C+\varepsilon e) \cap(-\operatorname{int} C)=\emptyset .
$$


By assumption, we know that $F(x, A)+C$ is a convex set. Using the separation theorem for convex sets, there exists some $g \in Y^{*} \mid\{0\}$, such that

$$
\inf \{g(F(x, y)+c+\varepsilon e): y \in A, c \in C\} \geq \sup \{g(-c): c \in C\} .
$$

From (3.2), we get $g \in C^{*} \backslash\{0\}$ and so

$$
g(F(x, y)) \geq-g(\varepsilon e) \text { for all } y \in A,
$$

where $e \in \operatorname{int} C$ with $g(e)>0$. Letting $f=\frac{g}{g(e)}$, then $f(F(x, y)) \geq-\varepsilon$, for all $y \in A$ and $f(e)=1$. Thus, $f \in C$ and so

$$
x \in \bigcup_{f \in C^{\prime}} V_{\varepsilon-f}(A, F) \text {. }
$$

This completes the proof.

Remark 3.1 When $\varepsilon=0$, it is easy to see that

$$
V_{\varepsilon-W}(A, F)=\bigcup_{f \in C^{\prime}} V_{\varepsilon-f}(A, F)=\bigcup_{f \in C * \backslash\{0\}} V_{\varepsilon-f}(A, F) .
$$

Therefore, Lemma 3.1 generalizes Lemma 2.1 in [27].

\section{Existence of the solutions}

Definition 4.1. The bifunction $F: A \times A \rightarrow Y$ is concave-like with respect to the first variable, if for $t \in[0,1]$, the following condition is satisfied: For $x_{1}, x_{2} \in A$, there exists $x_{3} \in A$, such that

$$
F\left(x_{3}, y\right) \in t F\left(x_{1}, y\right)+(1-t) F\left(x_{2}, y\right)+C, \quad \text { for all } y \in A .
$$

The bifunction $F: A \times A \rightarrow Y$ is convex-like with respect to the second variable, if for $t \in[0,1]$, the following condition is satisfied: for $y_{1}, y_{2} \in A$, there exists $y_{3} \in A$, such that

$$
F\left(x, y_{3}\right) \in t F\left(x, y_{1}\right)+(1-t) F\left(x, y_{2}\right)-C, \quad \text { for all } x \in A \text {. }
$$

Theorem 4.1. Let $A$ be a nonempty compact subset of $X$, and $f \in C^{\prime}$. Assume that the following conditions are satisfied:

(i) $F(x, x) \in C-\varepsilon e$, for all $x \in A$;

(ii) $F: A \times A \rightarrow Y$ is concave-like with respect to the first variable and convex-like with respect to the second variable;

(iii) For each fixed $y \in A$, the function $x \mapsto f(F(x, y))$ is upper semicontinuous on $A$. Then, $V_{\varepsilon-f}(A, F) \neq \varnothing$.

Proof. Define the set-valued map $G: A \rightarrow 2^{A}$ by

$$
G(y)=\{x \in A, f(F(x, y)) \geq-\varepsilon\}
$$

By assumption, $y \in G(y)$, for all $y \in A$, so $G(y) \neq \varnothing$. By assumption, we can see that $G(y)$ is a closed subset of $A$. Next, we prove that $\cap\{G(y): y \in A\} \neq \varnothing$. Since $A$ is a compact, we need to show that $\bigcap_{i=1}^{n} G\left(y_{i}\right) \neq \emptyset$ for any arbitrary chosen $y_{1}, \ldots, y_{n}$ in $A$. Suppose it is not true. Then, there exists a set $B=\left\{y_{1}, \ldots, y_{n}\right\} \subset A$ such that $\bigcap_{i=1}^{n} G\left(y_{i}\right) \neq \emptyset$. Thus, for any $x \in A$, there exists $y_{i} \in B$ such that $x \notin G\left(y_{i}\right)$. It follows 
that

$$
f\left(F\left(x, y_{i}\right)\right)+\varepsilon<0
$$

and so there exists $\eta_{i}>0$ such that

$$
f\left(F\left(x, y_{i}\right)\right)+\varepsilon<-\eta_{i}, \quad i=1,2, \ldots, n .
$$

Since $x \mapsto f(F(x, y))$ is upper semicontinuous on $A$, we can choose $\eta>0$ such that, for any $x \in A$, there exists $y_{i} \in B$ satisfying

$$
f\left(F\left(x, y_{i}\right)\right)+\varepsilon+\eta<0 .
$$

Define $g: A \rightarrow R^{n}$ by

$$
g(x)=\left(-f\left(F\left(x, y_{1}\right)\right)-\varepsilon-\eta,-f\left(F\left(x, y_{2}\right)\right)-\varepsilon-\eta, \ldots,-f\left(F\left(x, y_{n}\right)\right)-\varepsilon-\eta\right),
$$

where $x \in A$. We get

$$
g(x) \notin \operatorname{int} R_{+}^{n}, \quad \text { for all } x \in A .
$$

Since $f \in C^{\prime}$ and $F(x, y)$ is concave-like with respect to the first variable, we can see that, for $t \in[0,1], x_{1}, x_{2} \in A$, there exists $x_{3} \in A$ such that

$$
g\left(x_{3}\right) \in \operatorname{tg}\left(x_{1}\right)+(1-t) g\left(x_{2}\right)-C .
$$

This shows that $g(A)+R_{+}^{n}$ is a convex set. It follows from (4.1) that

$$
0 \notin g(A)+\operatorname{int} R_{+}^{n} \text {. }
$$

By the separation theorem of convex sets (see, for example, [34]), we can find $t_{1}, \ldots, t_{n}$ $\geq 0$ with $\sum_{i=1}^{n} t_{i}=1$ such that

$$
0 \leq \sum_{i=1}^{n} t_{i}\left(-f\left(F\left(x, y_{i}\right)\right)-\varepsilon-\eta\right), \text { for all } x \in A
$$

It follows that

$$
\sum_{i=1}^{n} t_{i} f\left(F\left(x, y_{i}\right)\right) \leq-\varepsilon-\eta, \text { for all } x \in A .
$$

By assumption, there exists $y \in A$ such that

$$
F(x, y) \in \sum_{i=1}^{n} t_{i}\left(F\left(x, y_{i}\right)\right)-C, \text { for all } x \in A .
$$

Since $f \in C$, we have

$$
f(F(x, y)) \leq f\left(\sum_{i=1}^{n} t_{i}\left(F\left(x, y_{i}\right)\right)\right), \text { for all } x \in A
$$

So $f(F(x, y)) \leq-\varepsilon-\eta<-\varepsilon$, for all $x \in A$. Setting $x=y$, it follows that

$$
f(F(y, y))<-\varepsilon .
$$


On the other hand, by the assumption,

$$
f(F(y, y)) \geq-\varepsilon .
$$

This is a contradiction. Therefore, $\cap\{G(y): y \in A\} \neq \varnothing$, and so there exists $x \in \cap\{G$ $(y): y \in A\}$. This means that $V_{\varepsilon-f}(A, F) \neq \varnothing$. This completes the proof.

\section{Connectedness of the solutions set}

In this section, we discuss the connected results of $\varepsilon$-weak efficient solutions set and $\varepsilon$ efficient solutions set.

Definition 5.1. Let $A$ be a convex set. The bifunction $F: A \times A \rightarrow Y$ is $C$-concave with respect to the first variable, if for $t \in[0,1], x_{1}, x_{2} \in A$,

$$
F\left(t x_{1}+(1-t) x_{2}, y\right) \in t F\left(x_{1}, y\right)+(1-t) t F\left(x_{2}, y\right)+C .
$$

It is clear that when $A$ is a convex set, $F: A \times A \rightarrow Y$ is $C$-concave with respect to the first variable, then it is concave-like about the first variable.

Theorem 5.1 Let $A$ be a nonempty compact convex subset of $X$, and $f \in C^{\prime}$. Assume that the following conditions are satisfied.

(i) $F(x, x) \in C-\varepsilon e$, for all $x \in A$;

(ii) $F: A \times A \rightarrow Y$ is $C$-concave with respect to the first variable and convex-like with respect to the second variable;

(iii) For each fixed $y \in A$, the function $x \mapsto f(F(x, y))$ is upper semicontinuous on $A$;

(iv) $D=\{F(x, y): x, y \in A\}$ is a bounded set of $Y$.

Then, $V_{\varepsilon-W}(A, F)$ is a connected set.

Proof. Define a set-valued mapping $H: C \rightarrow 2^{A}$ by

$$
H(f)=V_{\in-f}(A, F), f \in C^{\prime} .
$$

By Theorem 4.1, we know that, for each $f \in C, V_{\varepsilon-f}(A, F) \neq \varnothing$. It is easy to see that $C^{\prime}$ is a convex set and so is connected. Next, we prove that, for each $f \in C^{\prime}, H(f)$ is a connected set. Let $x_{1}, x_{2} \in H(f)$, we have $x_{1}, x_{2} \in A$, and

$$
f\left(F\left(x_{i}, y\right)\right) \geq-\varepsilon, \quad \forall y \in A, i=1,2 .
$$

Because $F: A \times A \rightarrow Y$ is $C$-concave with respect to the first variable, for each fixed $y$ $\in A, t \in[0,1]$,

$$
F\left(t x_{1}+(1-t) x_{2}, y\right) \in t F\left(x_{1}, y\right)+(1-t) F\left(x_{2}, y\right)+C .
$$

Hence

$$
t f\left(F\left(x_{1}, y\right)\right)+(1-t) f\left(F\left(x_{2}, y\right)\right) \leq f\left(F\left(t x_{1}+(1-t) x_{2}, y\right)\right) .
$$

It follows from (5.1) that

$$
f\left(F\left(t x_{1}+(1-t) x_{2}, y\right)\right) \geq-\varepsilon .
$$

Hence

$$
t x_{1}+(1-t) x_{2} \in H(f)
$$

and so $H(f)$ is a convex set. Thus, it is a connected set. 
Next, we show that $H$ is upper semicontinuous on $C$. Since $A$ is compact, we only need to show that $H$ is closed. Let $\left\{\left(f_{\alpha}, x_{\alpha}\right): \alpha \in I\right\} \subset \operatorname{Graph}(H)$ be a net such that $\left(f_{\alpha}\right.$, $\left.x_{\alpha}\right) \rightarrow(f, x)$, where $f_{\alpha} \rightarrow f$ means that $\left\{f_{\alpha}\right\}$ converges to $f$ with respect to the strong topology $\beta\left(Y^{*}, Y\right)$ in $Y^{* *}$. Since $C^{\prime}$ is a closed set and $A$ is a compact set, we know that $(f, x) \in C \times A$. From $x_{\alpha} \in H\left(f_{\alpha}\right)=V_{\varepsilon-} f \alpha(A, F)$, we have

$$
f_{\alpha}\left(F\left(x_{\alpha}, y\right)\right) \geq-\varepsilon
$$

For any $\delta>0$,

$$
U=\left\{\gamma^{*} \in Y^{*}: \sup _{u \in D}\left|y^{*}(u)\right|<\delta\right\}
$$

is a neighborhood of 0 with respect with to $\beta\left(Y^{*}, Y\right)$. Since $f_{\alpha} \rightarrow f$, there exists $\alpha_{0} \in$ $I$ such that $f_{\alpha}-f \in U$, for all $\alpha \geq \alpha_{0}$. It follows that

$$
\sup _{u \in D}\left|\left(f_{\alpha}-f\right)(u)\right|<\delta, \quad \text { whenever } \alpha \geq \alpha_{0} .
$$

Therefore, for any $y \in A$,

$$
\left|\left(f_{\alpha}-f\right)\left(F\left(x_{\alpha}, y\right)\right)\right|<\delta, \quad \text { whenever } \alpha \geq \alpha_{0}
$$

and so

$$
\lim _{\alpha}\left(f_{\alpha}\left(F\left(x_{\alpha}, y\right)\right)-f\left(F\left(x_{\alpha}, y\right)\right)\right)=0, \quad \text { for all } y \in A .
$$

Because $x \mapsto f(F(x, y))$ is upper semicontinuous on $A$, then

$$
\lim \sup f\left(F\left(x_{\alpha}, y\right)\right) \leq f(F(x, y)) .
$$

From (5.2), (5.3) and (5.4), we have

$$
\begin{aligned}
-\varepsilon & \leq \limsup _{\alpha}\left(F\left(x_{\alpha}, y\right)\right) \\
& \leq \lim _{\alpha}\left(f_{\alpha}\left(F\left(x_{\alpha}, y\right)\right)-f\left(F\left(x_{\alpha}, y\right)\right)\right)+\lim \sup f\left(F\left(x_{\alpha}, y\right)\right) \\
& \leq f(F(x, y)) .
\end{aligned}
$$

Hence, $x \in H(f)=V_{\varepsilon-f}(A, F)$. By Theorem 3.1 in [35], $\bigcup_{f \in C^{\prime}} V_{\varepsilon-f}(A, F)$ is a connected set. Because $F: A \times A \rightarrow Y$ is convex-like with respect to the second variable, we have $F(x, A)+C$ is a convex set, by Lemma 3.1,

$$
V_{\varepsilon-W}(A, F)=\bigcup_{f \in C^{\prime}} V_{\varepsilon-f}(A, F)
$$

is a connected set. This completes the proof.

Next, we give an example to illustrate Theorem 5.1.

Example 5.1 Let $X=R, Y=R^{2}, C=R_{+}^{2}=\left\{\left(x_{1}, x_{2}\right): x_{1} \geq 0, x_{2} \geq 0\right\}, \varepsilon=2, e=(2,3)$, and $A=[0,2]$. Let

$$
F(x, y)=\left(y-x^{2}-2, y-x^{2}-4\right), \quad \forall x, y \in A .
$$

Then, F satisfies all conditions of Theorem 5.1. It is easy to see that $V_{\varepsilon-W}(A, F)=[0, \sqrt{2}]$. Clearly, $V_{\varepsilon-W}(A, F)$ is a nonempty connected set.

Definition 5.2. The bifunction $F: A \times A \rightarrow Y$ is $\varepsilon-C$ strictly monotone if, for any $x, y$ $\in A, x \neq y$, 


$$
F(x, y)+F(y, x) \in-\text { int } C-2 \varepsilon e .
$$

Theorem 5.2 Suppose that all conditions of Theorem 5.1 are satisfied and $F: A \times A$ $\rightarrow Y$ is $\varepsilon-C$ strictly monotone. Then, $V_{\varepsilon-W}(A, F)$ is a path connected set.

Proof. Define the set-valued mapping $H: C^{\prime} \rightarrow 2^{A}$ by

$$
H(f)=V_{\varepsilon-f}(A, F), f \in C^{\prime} .
$$

By Theorem 4.1, we know that, for each $f \in C, V_{\varepsilon-f}(A, F) \neq \varnothing$. Furthermore, because $F: A \times A \rightarrow Y$ is $\varepsilon-C$ strictly monotone, it is easy to see that, for each $f \in C^{\prime}, H(f)=$ $V_{\varepsilon-f}(A, F)$ is a single point set. From the proof of Theorem 5.1, we know that $H$ is upper semicontinuous on $C$ and so it is continuous on $C$. Since $C$ is a convex set, so it is a path connected set. Hence,

$$
V_{\varepsilon-W}(A, F)=H\left(C^{\prime}\right)=\bigcup_{f \in C^{\prime}} H(f)=\bigcup_{f \in C^{\prime}} V_{\varepsilon-f}(A, F)
$$

is a path connected set. This completes the proof.

Next, we give an example to illustrate Theorem 5.2.

Example 5.2 Let $X=R, Y=R^{2}, C=R_{+}^{2}=\left\{\left(x_{1}, x_{2}\right): x_{1} \geq 0, x_{2} \geq 0\right\}, \varepsilon=-1, e=(1$, 2), and $A=[-1,1]$. Let

$$
F(x, y)=(x(y-x)+1, x(y-x)+2), \quad \forall x, y \in A .
$$

Then, $F$ satisfies all the conditions of Theorem 5.2. It is easy to see that $V_{\varepsilon-W}(A, F)=$ $\{0\}$. Clearly, $V_{\varepsilon-W}(A, F)$ is a nonempty path connected set.

Next, we give a lemma before we give the connectedness theorem of $\varepsilon$-efficient solutions set.

Lemma 5.1. Suppose that all conditions of Theorem 5.2 are satisfied, then

$$
\bigcup_{f \in C^{\prime \prime}} V_{\varepsilon-f}(A, F) \subset V_{\varepsilon}(A, F) \subset c l\left(\bigcup_{f \in C^{\prime \prime}} V_{\varepsilon-f}(A, F)\right) .
$$

Proof. By Theorem 4.1, we know that, for each $f \in C^{\prime}, V_{\varepsilon-f}(A, F) \neq \varnothing$. By definition, we have

$$
\bigcup_{f \in C^{\prime}} V_{\varepsilon-f}(A, F) \subset V_{\varepsilon}(A, F) \subset V_{\varepsilon-W}(A, F) .
$$

From Lemma 3.1, we have

$$
V_{\varepsilon-W}(A, F)=\bigcup_{f \in C^{\prime}} V_{\varepsilon-f}(A, F) .
$$

By (5.5) and (5.6), we have

$$
\bigcup_{f \in C^{\prime \prime}} V_{\varepsilon-f}(A, F) \subset V_{\varepsilon}(A, F) \subset \bigcup_{f \in C^{\prime}} V_{\varepsilon-f}(A, F) .
$$

Next, we prove that

$$
\bigcup_{f \in C^{\prime}} V_{\varepsilon-f}(A, F) \subset c l\left(\bigcup_{f \in C^{\prime \prime}} V_{\varepsilon-f}(A, F)\right) .
$$

Define the set-valued mapping: $H: C^{\prime} \rightarrow 2^{A}$ by

$$
H(f)=V_{\varepsilon-f}(A, F), f \in C^{\prime} .
$$


From Theorem 5.2, we know that $H(f)$ is a single-valued mapping, and $H$ is continuous on $C$.

Let $x \in \bigcup_{f \in C^{\prime}} V_{\varepsilon-f}(A, F)$. Then, there exists $f \in C^{\prime}$, such that

$$
\{x\}=V_{\varepsilon-f}(A, F)=H(f) .
$$

Let $g \in C^{\prime \prime}$, and set

$$
f_{n}=\frac{n-1}{n} f+\frac{1}{n} g=f+\frac{1}{n}(g-f) .
$$

Then, $f_{n} \in C^{\#}$ and $f_{n}(e)=1$. Thus, $f_{n} \in C^{u}$.

Next, we show that $\left\{f_{n}\right\}$ converges to $f$ with respect to the topology $\beta\left(Y^{*}, Y\right)$. For any neighborhood of 0 with respect to $\beta\left(Y^{*}, Y\right)$, there exist bounded subsets $B_{i} \in Y(i=1$, $2, \ldots, m)$ and $\delta>0$ such that

$$
\bigcap_{i=1}^{m}\left\{f \in Y^{*}: \sup _{y \in B_{i}}|f(y)|<\delta\right\} \subset U .
$$

Since $B_{i}$ is bounded and $g-f \in Y^{*}$, it is easy to see that $\left|(g-f)\left(B_{i}\right)\right|$ is bounded for $i$ $=1,2, \ldots, m$. This implies that there exists $N$ such that

$$
\sup _{\gamma \in B_{i}}\left|\frac{1}{n}(g-f)(\gamma)\right|<\delta, \quad i=1,2, \ldots, m ; \quad n \geq N .
$$

Hence, $\frac{1}{n}(g-f) \in U$, that is $f_{n}-f \in U$. Hence, $\left\{f_{n}\right\}$ converges to $f$ with respect to $\beta$ $\left(Y^{*}, Y\right)$.

Since $H$ is continuous on $f$, we have $H\left(f_{n}\right) \rightarrow H(f)$. Set $\left\{x_{n}\right\}=H\left(f_{n}\right)$, then

$$
\left\{x_{n}\right\}=H\left(f_{n}\right)=V_{\varepsilon-f_{n}}(A, F) \subset \bigcup_{f \in C^{\prime \prime}} V_{\varepsilon-f}(A, F) .
$$

Because $\{x\}=H(f)$, we have $x_{n} \rightarrow x$. This implies that

$$
x \in c l\left(\bigcup_{f \in C^{\prime \prime}} V_{\varepsilon-f}(A, F)\right) .
$$

Since $x \in \bigcup_{f \in C^{\prime}} V_{\varepsilon-f}(A, F)$ is arbitrary, we have

$$
\bigcup_{f \in C^{\prime}} V_{\varepsilon-f}(A, F) \subset c l\left(\bigcup_{f \in C^{\prime \prime}} V_{\varepsilon-f}(A, F)\right) .
$$

Therefore,

$$
\bigcup_{f \in C^{\prime \prime}} V_{\varepsilon-f}(A, F) \subset V_{\varepsilon}(A, F) \subset c l\left(\bigcup_{f \in C^{\prime \prime}} V_{\varepsilon-f}(A, F)\right) .
$$

This completes the proof.

Theorem 5.3. Suppose that all the conditions of Theorem 5.2 are satisfied. Then, $V_{\varepsilon}$ $(A, F)$ is a connected set.

Proof. By Lemma 5.1, we have

$$
\bigcup_{f \in C^{\prime \prime}} V_{\varepsilon-f}(A, F) \subset V_{\varepsilon}(A, F) \subset c l\left(\bigcup_{f \in C^{\prime \prime}} V_{\varepsilon-f}(A, F)\right) .
$$


From Theorem 5.2, we can get

$$
\bigcup_{f \in C^{\prime \prime}} V_{\varepsilon-f}(A, F)
$$

is connected set and so (5.7) implies that $V_{\varepsilon}(A, F)$ is a connected set. This completes the proof.

Remark 5.1 When $\varepsilon=0$, we can get

$$
\bigcup_{f \in C^{\prime \prime}} V_{\varepsilon-f}(A, F)=\bigcup_{f \in c \neq} V_{\varepsilon-f}(A, F) \text {. }
$$

Therefore, Theorem 5.3 generalizes Theorem 2.2 in [31].

Next, we give an example to illustrate Theorem 5.3.

Example 5.3 Let $X=R, Y=R, C=R_{+}, \varepsilon=1, e=1$, and $A=[1,2]$. Let $F(x, y)=x$ $(y-x)-1$ for all $x, y \in A$. Then, it is easy to check that all the conditions of Theorem 5.3 are satisfied and

$$
V_{\varepsilon}(A, F)=\{1\}
$$

Clearly, $V_{\varepsilon}(A, F)$ is a nonempty connected set.

\section{Abbreviations}

VEP: vector equilibrium problem.

\section{Acknowledgements}

This work was supported by the National Natural Science Foundation of China $(50874096,70831005)$ and the Open Fund (PLN0904) of State Key Laboratory of Oil and Gas Reservoir Geology and Exploitation (Southwest Petroleum University).

\section{Author details}

${ }^{1}$ Department of Mathematics, Sichuan University, Chengdu, Sichuan 610064, P.R. China ${ }^{2}$ State Key Laboratory of Oi and Gas Reservoir Geology and Exploitation, Southwest Petroleum University, Chengdu, Sichuan 610500, PR China ${ }^{3}$ Department of Applied Mathematics, Southwest Petroleum University, Chengdu, 610500, PR China

\section{Authors' contributions}

BC carried out the study of connectedness of $\varepsilon$-weak efficient and $\varepsilon$-efficient solutions sets for VEPs and drafted the manuscript. Q-YL participated in the design of the study. Z-BL gave some examples to show the main results. $\mathrm{N}$-JH conceived of the study, and participated in its design and coordination. All authors read and approved the final manuscript.

\section{Competing interests}

The authors declare that they have no competing interests.

Received: 27 October 2010 Accepted: 15 August 2011 Published: 15 August 2011

\section{References}

1. Blum, B, Oettli, W: From optimzation and variational inequalities to equilibrium problems. Math. Stud. 63, 123-145 (1994)

2. Chen, GY, Huang, XX, Yang, XQ: Vector Optimization: Set-Valued and Variational Analysis. Springer, Berlin (2005)

3. Giannessi, F, (ed): Vector Variational Inequilities and Vector Equilibria: Mathematical Theories. Kluwer, Dordrechet (2000)

4. Ansari, QH, Flores-Bazan, F: Recession methods for generalized vector equilibrium problems. J Math Anal Appl. 321, 132-146 (2006). doi:10.1016/j.jmaa.2005.07.059

5. Ansari, QH, Oettli, W, Schläger, D: A generalization of vector equilibrium. Math Methods Oper Res. 46, 147-152 (1997). doi:10.1007/BF01217687

6. Ansari, QH, Yao, JC: An existence result for the generalized vector equilibrium problem. Appl. Math. Lett. 12, 53-56 (1999)

7. Chen, B, Gong, $\mathrm{XH}$ : Continuity of the solution set to parametric set-valued weak vector equilibrium problems. Pacific J. Optim. 6, 511-520 (2010)

8. Chen, CR, Li, SJ, Teo, KL: Solution semicontinuity of parametric generalized vector equilibrium problems. J. Glob. Optim. 45, 309-318 (2009). doi:10.1007/s10898-008-9376-9

9. Konnov, IV, Yao, JC: Existence of solutions of generalized vector equilibrium problems. J. Math. Anal. Appl. 233, 328-335 (1999). doi:10.1006/jmaa.1999.6312

10. Fu, JY: Generalized vector quasivariational problems. Math. Methods Oper. Res. 52, 57-64 (2000). doi:10.1007/ s001860000058 
11. Hou, SH, Yu, H, Chen, GY: On vector quasi-equilibrium problems with set-valued maps. J. Optim. Theory Appl. 119, 139-154 (2003)

12. Tan, NX: On the existence of solutions of quasi-variational inclusion problems. J. Optim. Theory Appl. 123, 619-638 (2004). doi:10.1007/s10957-004-5726-z

13. Peng, JW, Joseph Lee, HW, Yang, XM: On systems of generalized vector quasi-quilibrium problem with set-valued maps. J. Glob. Optim. 35, 139-158 (2006)

14. Lin, LJ, Ansari, QH, Huang, YJ: Some existence results for solutions of generalized vector quasi-equilibrium problems. Math. Methods Oper. Res. 65, 85-98 (2007). doi:10.1007/s00186-006-0102-4

15. Long, XJ, Huang, NJ, Teo, KL: Existence and stability of solutions for generalized strong vector quasi-equilibrium problem. Math. Comput. Model. 47, 445-451 (2008). doi:10.1016/j.mcm.2007.04.013

16. Huang, NJ, Li, J, Yao, JC: Gap functions and existence of solutions for a system of vector equilibrium problems. J. Optim. Theory Appl. 133, 201-212 (2007). doi:10.1007/s10957-007-9202-4

17. Li, J, Huang, NJ: Implicit vector equilibrium problems via nonlinear scalarisation. Bull. Aust. Math. Soc. 72, 161-172 (2005). doi:10.1017/S000497270003495X

18. Li, J, Huang, NJ, Kim, JK: On implicit vector equilibrium problems. J. Math. Anal. Appl. 283, 501-512 (2003). doi:10.1016/ S0022-247X(03)00277-4

19. Park, S: Fixed points of better admissible maps on generalized convex spaces. J. Korea Math. Soc. 37(6), 885-899 (2000)

20. Park, S: Equilibrium existence theorems in KKM spaces. Nonlinear Anal. TMA. 69, 4352-4364 (2008). doi:10.1016/j. na.2007.10.058

21. Park, S: Compact Browder maps and equilibria of abstract economies. J. Appl. Math. Comput. 26, 555-564 (2008). doi:10.1007/s12190-007-0022-3

22. Park, S: Remarks on fixed points, maximal elements, and equilibria of economies in abstract convex spaces. Taiwanese J. Math. 12, 1365-1383 (2008)

23. Park, S, Kim, H: Coincidence theorems for admissible multifunctions on generalized convex spaces. J. Math. Anal. Appl. 197, 173-187 (1996). doi:10.1006//maa.1996.0014

24. Park, S, Kim, H: Founditions of the KKM theory on generalized convex spaces. J. Math. Anal. Appl. 209, 551-571 (1997). doi:10.1006/jmaa.1997.5388

25. Lee, GM, Kim, DS, Lee, BS, Yen, ND: Vector variational inequality as a tool for studying vector optimization problems. Nonlinear Anal.: Theory, Methods Appl. 34, 745-765 (1998). doi:10.1016/S0362-546X(97)00578-6

26. Cheng, YH: On the connectedness of the solution set for the weak vector variational inequality. J. Math. Anal. Appl. 260, 1-5 (2001). doi:10.1006/jmaa.2000.7389

27. Gong, XH: Efficiency and Henig efficiency for vector equilibrium problems. J. Optim. Theory Appl. 108, 139-154 (2001). doi:10.1023/A:1026418122905

28. Gong, XH, Fu, WT, Liu, W: Super efficiency for a vector equilibrium in locally convex topological vector spaces. In: Giannessi F (ed.) Vector Variational Inequalities and Vector Equilibria: Mathematical Theories. pp. 233-252. Kluwer Academic Publishers, Netherlands (2000)

29. Gong, XH: Connectedness of the solution sets and scalarization for vector equilibrium problems. J. Optim. Theory Appl. 133, 151-161 (2007). doi:10.1007/s10957-007-9196-y

30. Chen, B, Gong, XH, Yuan, SM: Connectedness and compactness of weak efficient solutions set for set-valued vector equilibrium problems. J. Inequal. Appl 2008, 15 (2008). Article ID 581849

31. Gong, XH, Yao, JC: Connectedness of the set of efficient solutions for generalized systems. J. Optim. Theory Appl. 138, 189-196 (2008). doi:10.1007/s10957-008-9378-2

32. Zhong, RY, Huang, NJ, Wong, MM: Connectedness and path-connectedness of solution sets for symmetric vector equilibrium problems. Taiwan. J. Math. 13, 821-836 (2009)

33. Aubin, JP, Ekeland, I: Applied Nonlinear Analysis. Wiley, New York (1984)

34. Robertson, AP, Robertson, W: Topological Vector Spaces. Cambridge University Press (1964)

35. Warburton, AR: Quasi-concave vector maximization: connectedness of the sets of Pareto-optimal and weak Paretooptimal alternatives. J. Optim. Theory Appl. 40, 537-557 (1983). doi:10.1007/BF00933970

doi:10.1186/1687-1812-2011-36

Cite this article as: Chen et al: Connectedness of approximate solutions set for vector equilibrium problems in Hausdorff topological vector spaces. Fixed Point Theory and Applications 2011 2011:36.

\section{Submit your manuscript to a SpringerOpen ${ }^{\circ}$ journal and benefit from:}

- Convenient online submission

- Rigorous peer review

- Immediate publication on acceptance

- Open access: articles freely available online

- High visibility within the field

- Retaining the copyright to your article

Submit your next manuscript at $\gg$ springeropen.com 